\title{
The spatial distribution of stars in open clusters
}

\author{
Néstor Sánchez and Emilio J. Alfaro \\ Instituto de Astrofísica de Adalucía-CSIC, Apdo. 3004, E-18080, Granada, Spain \\ email: [nestor, emilio] @iaa.es
}

\begin{abstract}
We study the internal spatial structure of 16 open clusters in the Milky Way spanning a wide range of ages. For this, we use the minimum-spanning-tree method (the $Q$ parameter, which enables one to classify the stellar distribution as either radially or fractally clustered), King-profile fitting, and the correlation dimension $\left(D_{\mathrm{c}}\right)$ for those clusters with fractal patterns. On average, clusters with fractal-like structure are younger than those exhibiting radial stellardensity profiles. There is a significant correlation between $Q$ and the cluster age measured in crossing-time units. For fractal clusters, there is a significant correlation between fractal dimension and age. These results support the idea that stars in newly born clusters likely follow the fractal patterns of their parent molecular clouds, and eventually evolve towards more centrally concentrated structures. However, stellar clusters as old as $\sim 100 \mathrm{Myr}$ can exist that have not totally destroyed their fractal structure. Finally, we have found the intriguing result that the lowest fractal dimensions obtained for the open clusters seem to be considerably smaller than the average value measured in Galactic molecular cloud complexes.
\end{abstract}

Keywords. ISM: structure, methods: statistical, open clusters and associations: general, stars: formation

\section{Introduction}

The hierarchical structure observed in some open clusters is presumably a consequence of their formation in a turbulent medium with an underlying fractal structure (Elmegreen \& Scalo 2004). Otherwise, open clusters having central stellar concentrations with radial stellar-density profiles likely reflect the dominant role of gravity, either on the primordial gas structure or as a result of rapid evolution from a more structured state (Lada \& Lada 2003). Therefore, an analysis of the stellar distribution may yield information on the formation process and early evolution of open clusters. It is necessary, however, that this kind of analysis is done by measuring the cluster structure in an objective, quantitative, and systematic way. Here, we study the internal spatial structure in a sample of 16 open clusters spanning a wide range of ages.

\section{Procedure}

1. We first used VIzIER (Ochsenbein et al. 2000) to search for catalogs containing both positions and proper motions of stars in open cluster regions.

2. We applied a robust nonparametric method to assign cluster membership (Cabrera-Caño \& Alfaro 1990). This method makes no a priori assumptions about cluster and field-star distributions.

3. We fitted King (1962) profiles to the radial density distribution of cluster members. From these fits, we obtained both the core $\left(R_{\mathrm{c}}\right)$ and tidal radius $\left(R_{\mathrm{t}}\right)$.

4. Next, we used the minimum-spanning-tree technique (see Figure 1) to calculate the dimensionless parameter $Q$ (see details in Cartwright \& Whitworth 2004 and Schmeja \& 
IC 2391

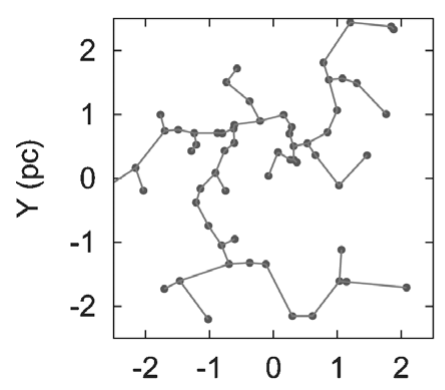

M 34

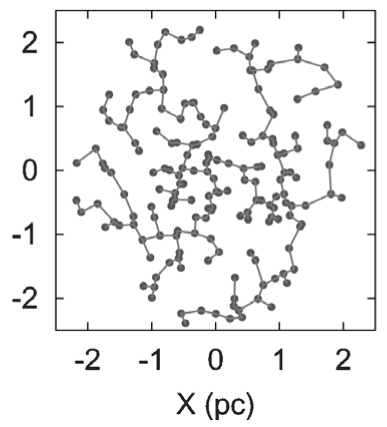

M 11

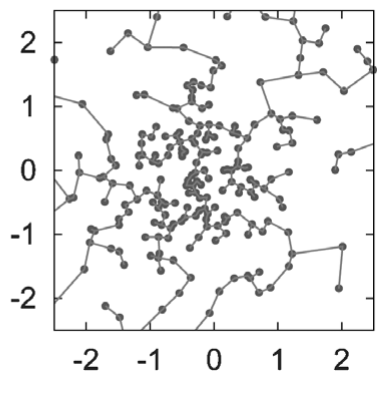

Figure 1. The minimum spanning tree is the set of straight lines connecting the points such that the sum of their lengths is at minimum. Here, we show minimum spanning trees for three open clusters, from which we can calculate the structure parameter $Q$. Star positions are indicated by blue circles and red lines represent the tree. The value of $Q$ quantifies the stellar spatial distribution. In IC 2391 the stars are distributed following an irregular fractal pattern $(Q=0.77<0.8)$, in M 34 they are distributed roughly homogeneously $(Q=0.8)$, and in M 11 the stars follow a radial density profile $(Q=1.02>0.8)$.

Table 1. Properties of the sample clusters.

\begin{tabular}{lcrrrrrc}
\hline Name & $\log T$ & $D$ & $N_{\mathrm{s}}$ & $R_{\mathrm{c}}$ & $R_{\mathrm{t}}$ & $Q$ & $D_{\mathrm{c}}$ \\
\hline IC 2391 & 7.661 & 175 & 62 & 1.46 & 2.65 & 0.77 & $1.74 \pm 0.20$ \\
M 11 & 8.302 & 1877 & 289 & 1.98 & 4.49 & 1.02 & $\ldots$ \\
M 34 & 8.249 & 499 & 181 & 0.11 & 1.73 & 0.80 & $2.04 \pm 0.05$ \\
M 67 & 9.409 & 908 & 354 & 2.21 & 5.92 & 0.98 & $\ldots$ \\
NGC 188 & 9.632 & 2047 & 1459 & 2.90 & 10.57 & 0.91 & $\ldots$ \\
NGC 581 & 7.336 & 2194 & 526 & 1.38 & 11.86 & 0.76 & $1.79 \pm 0.06$ \\
NGC 1513 & 8.110 & 1320 & 156 & 1.55 & 7.73 & 0.72 & $1.82 \pm 0.09$ \\
NGC 1647 & 8.158 & 540 & 683 & 1.23 & 8.86 & 0.70 & $1.94 \pm 0.02$ \\
NGC 1817 & 8.612 & 1972 & 277 & 3.39 & 11.97 & 0.79 & $1.94 \pm 0.04$ \\
NGC 1960 & 7.468 & 1318 & 311 & 2.96 & 8.77 & 0.87 & $\ldots$ \\
NGC 2194 & 8.515 & 3781 & 228 & 3.17 & 10.31 & 0.85 & $\ldots$ \\
NGC 2548 & 8.557 & 769 & 168 & 2.61 & 9.16 & 0.90 & $\ldots$ \\
NGC 4103 & 7.393 & 1632 & 799 & 0.72 & 10.74 & 0.78 & $1.85 \pm 0.04$ \\
NGC 4755 & 7.216 & 1976 & 196 & 1.11 & 3.50 & 0.94 & $\ldots$ \\
NGC 5281 & 7.146 & 1108 & 80 & 0.62 & 2.44 & 0.84 & $\ldots$ \\
NGC 6530 & 6.867 & 1330 & 145 & 1.43 & 7.47 & 0.67 & $1.74 \pm 0.09$ \\
\hline
\end{tabular}

$T$ : cluster age $(\mathrm{Myr}), D$ : distance $(\mathrm{pc}), N_{\mathrm{s}}$ : number of members, $R_{\mathrm{c}}$ : core radius $(\mathrm{pc}), R_{\mathrm{t}}$ : tidal radius (pc), $Q$ : structure parameter, $D_{\mathrm{c}}=$ correlation dimension.

Klessen 2006). The value $Q \simeq 0.8$ separates radial $(Q>0.8)$ from fractal-type clustering $(Q<0.8)$.

5. Finally, we calculated the correlation dimension $\left(D_{c}\right)$ and its associated uncertainty by employing an algorithm which gives reliable results (Sánchez et al. 2007a; Sánchez \& Alfaro 2008).

\section{Main results}

Table 1 summarizes the relevant data (ages and distances were taken from the WEBDA database). 


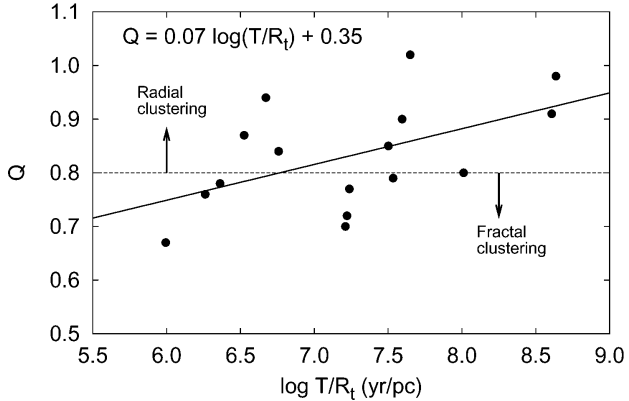

Figure 2. Structure parameter $Q$ as a function of logarithmic age divided by the tidal radius, which is nearly proportional to age in crossing-times units. The dashed line at $Q=0.8$ roughly separates radial from fractal clustering. The best linear fit (equation at the top) is represented by a solid line.

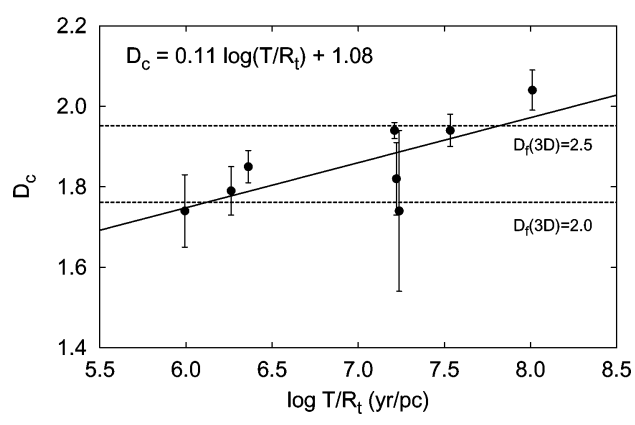

Figure 3. Calculated correlation dimension as a function of age (in crossing-time units). The best linear fit (equation at the top) is represented by a solid line. For reference, horizontal dashed lines indicate the values corresponding to three-dimensional distributions with fractal dimensions of $D_{\mathrm{f}}=2.0$ and 2.5 .

On average, stars in young clusters tend to be distributed following clustered, fractallike patterns $(Q<0.8)$, while older clusters tend to exhibit radial stellar-density profiles $(Q>0.8)$. However, the statistical analysis indicates that there is no significant correlation between $Q$ and $\log (T)$. If, instead, we consider the variable $T / R_{\mathrm{t}}$, which is proportional to the cluster age measured in crossing-time units (assuming nearly the same typical velocity dispersion for the open clusters), a significant correlation is observed (Figure 2). We also observe significant correlations (confidence levels $>96 \%$ ) between $D_{\mathrm{c}}$ and $T$ (cluster age) and also between $D_{\mathrm{c}}$ and $T / R_{\mathrm{t}}$ (age in crossing-time units) for those clusters with internal substructure (Figure 3).

\section{Discussion}

Our results support the idea that stars in newly born clusters likely follow the fractal patterns of their parent molecular clouds, and that they eventually evolve towards more centrally concentrated structures (see Schmeja \& Klessen 2006; Schmeja et al. 2008, 2009; Sánchez et al. 2007a, 2009). However, this seems to be only an overall trend. The very young cluster $\sigma$ Orionis (age $\sim 3 \mathrm{Myr}$ ) exhibits a radial density gradient with $Q \simeq 0.88$ (Caballero 2008). On the other hand, Table 1 shows open clusters as old as $\sim 100 \mathrm{Myr}$ that have not totally destroyed their clumpy structure (for example, both NGC 1513 and NGC 1647 have $Q \sim 0.7$ ). Goodwin \& Whitworth (2004) simulated the dynamical evolution of young clusters and showed that survival of the initial substructure depends strongly on the initial velocity dispersion. Fractal clusters with a low velocity dispersion tend to erase their substructure rather quickly. However, if the velocity dispersion is high, such that the cluster remains supported against its own gravity or even expands, significant levels of substructure can survive for several crossing times. Thus, our results give some observational support to Goodwin \& Whitworth's (2004) simulations.

From Figure 3, we can see that clusters with the smallest correlation dimensions $\left(D_{\mathrm{c}}=1.74\right)$ would have three-dimensional fractal dimensions around $D_{\mathrm{f}} \sim 2.0$ (estimated from previous papers; see, e.g., figure 1 in Sánchez \& Alfaro 2008). This is a very interesting result, because this value is considerably smaller than the average value estimated for Galactic molecular clouds in recent studies, which is $D_{\mathrm{f}} \simeq 2.6-2.7$ (Sánchez et al. 2005, 2007b). Young, newly born stars will probably reflect the conditions of the interstellar medium from which they formed. Therefore, a group of stars born from the 
same cloud, i.e., born at almost the same place and time, should have a fractal dimension similar to that of the parent cloud. If the fractal dimension of the interstellar medium has a nearly universal value around 2.6-2.7, then how can some clusters exhibit such small fractal dimensions? Perhaps some clusters develop some kind of substructure starting from an initially more homogeneous state. This possibility has been confirmed in numerical simulations (Goodwin \& Whitworth 2004), although some coherence in the initial velocity dispersion is required. Another explanation is that this difference is a consequence of a more clustered distribution of the densest gas from which stars form on the smallest spatial scales in the molecular cloud complexes, according to a multifractal scenario (Chappell \& Scalo 2001). Perhaps the star-formation process itself modifies in some (unknown) way the underlying geometry generating distributions of stars that can be very different from the distribution of gas in the parental clouds. Finally, one possibility is that the fractal dimension of the interstellar medium in the Galaxy does not have a universal value and therefore some regions form stars distributed following more clustered patterns. There is no a priori reason to assume that $D_{\mathrm{f}}$ has nearly the same value everywhere in the Galaxy, independent of either the dominant physical processes or environmental variables. Recent simulations of supersonic isothermal turbulence by Federrath et al. (2009) have shown that compressive forcing yields fractal dimensions for the interstellar medium significantly smaller $\left(D_{\mathrm{f}} \sim 2.3\right)$ compared to solenoidal forcing $\left(D_{\mathrm{f}} \sim 2.6\right)$. Thus, $D_{\mathrm{f}}$ could be very different from region to region in the Galaxy depending on the main physical processes driving the turbulence. At least on galactic scales, it has been shown that there are significant differences in the fractal dimension of the distribution of star-forming sites among galaxies, contrary to the universal picture previously claimed in the literature (see Sánchez \& Alfaro 2008), so that the possibility of a nonuniversal fractal dimension for the interstellar medium in the Galaxy cannot, in principle, be ruled out.

\section{References}

Caballero, J. A. 2008, MNRAS, 383, 375

Cabrera-Caño, J. \& Alfaro, E. J. 1990, A\& A, 235, 94

Cartwright, A. \& Whitworth, A. P. 2004, MNRAS, 348, 589

Chappell, D. \& Scalo, J. 2001, ApJ, 551, 712

Elmegreen, B. G. \& Scalo, J. 2004, ARA\&A, 42, 211

Federrath, C., Klessen, R. S., \& Schmidt, W. 2009, ApJ, 692, 364

Goodwin, S. P. \& Whitworth, A. P. 2004, A\&SA, 413, 929

King, I. 1962, $A J, 67,471$

Lada, C. J. \& Lada, E. A. 2003, ARA\&A, 41, 57

Ochsenbein, F., Bauer, P., \& Marcout, J. 2000, A\&AS, 143, 221

Sánchez, N., Alfaro, E. J., \& Pérez, E. 2005, ApJ, 625, 849

Sánchez, N., Alfaro, E. J., Elias, F., Delgado, A. J., \& Cabrera-Caño, J. 2007a, ApJ, 667, 213

Sánchez, N., Alfaro, E. J., \& Pérez, E. 2007b, ApJ, 656, 222

Sánchez, N. \& Alfaro, E. J. 2008, ApJS, 178, 1

Sánchez, N. \& Alfaro, E. J. 2009, ApJ, 696, 2086

Schmeja, S. \& Klessen, R. S. 2006, A\&A, 449, 151

Schmeja, S., Kumar, M. S. N., \& Ferreira, B. 2008, MNRAS, 389, 1209

Schmeja, S., Gouliermis, D. A., \& Klessen, R. S. 2009, ApJ, 694, 367 